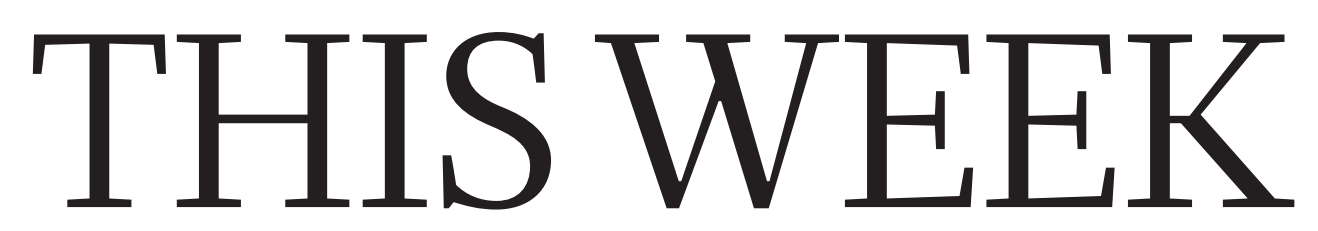

EDITORIALS

VACCINES Of past success and how to deliver on future promise $\mathbf{p . 4 2 0}$
WORLD VIEW How Europe can get the most for its Framework programme money $\mathbf{p . 4 2 1}$
PLUTO Carbon monoxide found around dwarf planet $\mathbf{p . 4 2 3}$

\title{
The long game
}

\section{Graphene is not a miracle material, just a very promising one. It will take restraint and sustained interest to deliver its potential.}

I $s$ it possible for a field of science to move too fast? Perhaps. Those who work on the form of carbon known as graphene have seen it rocket from the next big thing to a miracle material in less time than it can take for a research paper to be accepted and published. Yet although the hunt is on for applications that can exploit graphene's remarkable properties, the work necessary to find out how it could best be harnessed remains incomplete.

This is one reason why many scientists who attended a meeting on the subject earlier this month were cautious about raising expectations too high. 'Graphene: The Road to Applications' was held in Cambridge, Massachusetts, on 11-13 May, and was hosted by Nature Publishing Group.

As with most 'overnight' success stories, the field of graphene research actually had a very slow start. Physicists have long been fascinated by this one-atom-thick planar crystal of carbon atoms, but until a few years ago most considered it a hypothetical system, useful for studying fundamental properties of matter but not able to exist in free form. It took a steady build-up of theoretical work and pioneering experiments over several decades before the research storm broke when it became possible to isolate graphene sheets in a laboratory.

The material certainly has an impressive set of properties. And there is no denying its tantalizing promise. As a thin membrane flexible, strong and impermeable - graphene is an attractive platform on which to build devices. Add to that its high electron mobility and excellent optical and thermal properties, and the potential for applications seems endless. But the key word here is potential. The reality is that for many of graphene's widely promoted applications, it currently performs no better than existing materials and conventional approaches. For example, graphene performs relatively poorly as a transparent conductor in conventional displays, touch screens and photovoltaic cells. Neither is it a serious contender to replace silicon in standard electronics, because it does not work well as a digital switch, the essential function of a silicon transistor.

This should not necessarily be discouraging; the field is still very young. Those who talk up the potential of graphene's applications should remember that it typically takes any technology some 20 years to emerge from the lab and be commercialized - and even then it can succeed only with sustained effort and interest from researchers, industry and funding agencies (see Nature 469, 14-16; 2011).

It is crucial to identify and acknowledge practical hurdles - and when it comes to fabricating and designing graphene devices, there are many. As was stressed many times at the Cambridge meeting, the single biggest obstacle is the lack of a way to reliably produce high-quality graphene sheets in large quantities. The quality and structure of the sheets can differ from batch to batch, which can make devices behave inconsistently.

Furthermore, although the pristine form of graphene has high electron mobility, meaning that devices based on it have the potential to run at high speeds, this changes dramatically when it interacts with its environment, as it does when attached to a substrate. The material will be valuable only if it can perform reliably when put to work.

Graphene stands out from other materials for its combination of superlative properties. In the right application, it could have an any technology some 20 years to emerge from the lab and be commercialized." enormous impact. For example, although graphene does not perform well in digital electronics, there is much interest in using it to make high-frequency transistors for analogue applications - where it could be truly useful in wireless communication. Another important, if low-profile, role for graphene could be as a passive layer for heat management in electronic devices. Such targeting of graphene's potential will be vital to its success.

A few carefully chosen driver applications, although possibly not as obvious or exciting as some of the uses currently flagged, would give research on the material a better chance of holding on to wide interest in the long term - especially from funding agencies. Overnight success takes time to build. .
"It typically takes

\section{Copy and paste}

\section{A slow university investigation into serious accusations of misconduct benefits no one.}

A $s$ retractions go, it may not look like a big deal. Earlier this month, a statistics journal decided to pull a little-cited 2008 paper on the social networks of author-co-author relationships after it emerged that sections were plagiarized from textbooks and Wikipedia. The fact that this caused a wave of glee to ripple through the climate-change blogosphere takes some explaining.

Two of the paper's authors, Yasmin Said and Edward Wegman, both of George Mason University in Fairfax, Virginia, are also authors of an infamous 2006 report to Congress, co-written with statistician David Scott of Rice University in Houston, Texas. That report took aim at climatologist Michael Mann of Pennsylvania State University in University Park, suggesting that he was working in an isolated social network separated from "mainstream statisticians", and that he had such close ties with the rest of the field that truly independent peer review of his work was not possible. This report came to be known as the Wegman report, and has been frequently cited by climate-change sceptics.

This social-network analysis of Mann and his co-authors - with Mann's name removed - was cut down to an academic paper and published two years later in the journal Computational Statistics \& 